\section{Öko-Audit bei Stolzenberg - Einführung eines Umweltmanagementsystems}

Wie im Informationsdienst 6/94 schon berichtet, wurden vom IÖW zwei vom Berliner Senat für Wirtschaft und Technologie geförderte Öko-Audit-Modellprojekte durchgeführt. Über das erste Projekt bei der Fa. Märkisches Landbrot, das inzwischen fast abgeschlossen (die Zertifizierung steht noch aus) ist, wurde bereits berichtet. Hier sollen nun die ersten Ergebnisse des Projekts bei der Fa. Stolzenberg - Erfinder des Schnellhefters und Hersteller von Büroregistratursystemen wie Hefter, Mappen, Taschen mit verschiedenen Hängeregistratursystemen und Ordnungsmappen - vorgestellt werden.

Firmen aus der kartonverarbeitenden Branche verursachen $\mathrm{zwar}$ im Vergleich zu anderen Branchen relativ geringe direkte Belastungen für die Umwelt. Aber auch sie können in der Regel durch systematische Untersuchungen noch Optimierungspotentiale finden. Typische Ansatzpunkte für die Branche liegen in der geeigneten Auswahl des Hauptrohstoffes Karton z.B. mit dem Einsatz von Recyclingkarton, bei dem Einkauf von umweltverträglichen Hilfsund Betriebsstoffen wie z.B. Druckfarben und Reinigungsmittel sowie in der Reduzierung des Verpackungsmaterials. Ein Bereich, der branchenunabhängig in fast jedem Unternehmen Verbesserungspotentiale - auch mit Kosteneinsparungen - verspricht, ist der Bereich Reststoffe und die damit verbundenen Entsorgungfragen.

\section{Projektablauf}

und Ergebnisse

Als erstes wurde eine Öko-Audit-Projektgruppe gegründet und eine für den organisatorischen Umweltschutz zuständige Person - Umweltschutzkoordinatorin - benannt. Die einzelnen Arbeitsschritte im Rahmen der Einführung des Umweltmanagementsystems sollten im Unternehmen regelmäßig in der Projektgruppe vorgestellt und diskutiert werden. So wurden zunächst in der Gruppe der Projektablauf erläutert und die Schwerpunkte für die erste Umweltprüfung festgelegt. Der Abfallbereich wurde von den Mitarbeitern als sehr wichtiger Punkt genannt, der im Rahmen der Umweltprüfung genauer untersucht werden sollte.

Mit dem nächsten Schritt wurde die Unternehmenspolitik in Form von Unternehmensleitlinien formuliert. Die Umweltleitlinien wurden von Mitarbeitern des Unternehmens zusammen mit der Geschäftsleitung erarbeitet und im Unternehmen diskutiert, bevor sie schließlich beschlossen wurden. Den oben angeführten branchentypischen Ansatzpunkten wurde u. a. mit den hier angeführten Grundsätzen Rechnung getragen.

\section{Die Umweltprüfung}

Für die erste Umweltprüfung verlangt die ÖkoAudit-Verordnung eine systematische und vollständige Erfassung aller für ein Unternehmen relevanten Umweltvorschriften. Wie bereits im Projekt beim Märkischen Landbrot festzustellen war, haben selbst Großunternehmen mit entsprechenden Stabsabteilungen in der Regel oft nur einen begrenzten Überblick über die für sie relevanten Umweltvorschriften. In der Regel fehlt den Unternehmen der systematische und vollständige Überblick über die für sie relevanten Vorschriften. Daher sind vor allem kleinere Unternehmen auf externe Unterstützung angewiesen. Aber auch intern geben zum Teil bereits vorliegende Unterlagen Auskunft über die einzuhaltenden Gesetze und Verordnungen.

Hinweise über relevante Vorschriften sind $\mathrm{zu}$ beziehen aus/von

- Sicherheitsdatenblättern der Lieferanten;

- im Unternehmen vorliegenden behördlichen Genehmigungen, Messungen u. ä.;

- einigen Fachverbänden und deren Informationsblättern;

- Industrie und Handelskammern: die IHK Nürnberg hat Leitfäden für einige Branchen mit Hinweisen über gesetzliche Anforderungen;

- zuständigen Behörden des Bundeslandes: sie können Nachfragen beantworten, ob und wann und in welcher Form Gesetze und Verordnungen für das Unternehmen relevant sind.

Mit der Erstellung einer Übersicht, die alle für das Unternehmen relevanten umweltrechtlichen Vorschriften mit Hinweisen über die betriebsspezifischen Bereiche umfaßt, wurde ein erster Schritt zum Aufbau des Umweltmanagementsystems geleistet. Darin wurden die Zuständigkeiten für die Einhaltung der Vorschriften und die Aktualisierung festgeschrieben und dokumentiert.

\section{Die Erfassung und Bewertung der Auswirkungen auf die Umwelt}

Der nächste Schritt im Rahmen der Umweltprüfung lag in der Erfassung und Bewertung der Auswirkungen auf die Umwelt. Die Stoffund Energieströme als Grundlage für die Erfassung der Wirkungen auf die Umwelt wurden nach dem inzwischen im IÖW bewährten Ansatz der betrieblichen Stoff- und Energiebilanz ermittelt.

Der betriebsindividuelle Öko-Kontenrahmen, der die Grundlage für die eigentliche Bilanz ist, wurde während eines Meetings gemeinsam mit dem Unternehmen erstellt. Die grobe Unterteilung nach Stoffen, Energien, Produkten und Emissionen bedurfte einer weiteren Differenzierung. Dabei war zu beachten, daß einerseits die Gruppierung der betrieblichen Stoff- und Energieströme artspezifisch erfolgt und andererseits vorhandene betriebliche Materialdatenstrukturen, Kontenpläne der Finanzbuchhaltung und interne Produkteinteilungen aufgegriffen und weitestgehend übernommen wurden. Die Nutzung dieser betrieblichen, teilweise DV-gestützten Unterlagen bilden mit die Gewähr dafür, daß in Zukunft die Erstellung der Stoffund Energiebilanz mit Hilfe des betrieblichen DV-Systems durchgeführt werden kann.

Abhängig von der Vielfalt der Inhaltsstoffe wurde die Gruppierung weiter unterteilt: So wurden die Rohstoffe nach den Materialien RecyclingKarton, TCF-Karton (Total chlorfreier Karton), sonstiger Karton, Metall und Gewebe gegliedert. Diese Differenzierung ermöglicht es zugleich, aus der mit dieser Struktur erstellten Stoff- und Energiebilanz leicht ökologische Aussagen zu treffen. Die Einteilung der unterschiedlichen Kartonsorten ermöglicht eine klare Aussage über den Recyclinganteil in dieser Stoffgruppe.

Wichtig ist bei der Erfassung, daß der Aufwand zur Erhebung der Mengeninformationen im Verhältnis zum erreichbaren Nutzen, dem Erkenntnisgewinn, gesehen wird. Die Ermittlung der Daten ist deshalb entsprechend der Umweltrelevanz der einzelnen Stoffe genau oder auch über Schätzungen zu bestimmen.

Unter Berücksichtigung der oben genannten Einschränkungen und der Tatsache, daß bei der Bilanzierung Lagerbestände und eingegangene Rohstoffverpackungen nicht erfaßt wurden, war eine Beurteilung der Umweltauswirkungen auf Grundlage der erstellten Bilanz möglich, da umweltrelevante Stoffströme nicht vernachlässigt wurden.

\section{Beurteilung der erfaßten Stoff- und Energieströme}

Umweltprobleme ergeben sich nicht aus der puren Anwesenheit von Stoffen in der Umwelt, sondern durch ihre Wirkungen auf den Menschen (z. B. Toxizität, Ozonschichtzerstörung, Eutrophierung und Vergiftung der Gewässer etc.). Es wurden vier zu bewertende Gruppen unterschieden, die unterschiedliche Bewertungsansätze erfordern:

- Roh-, Hilfs- und Betriebsstoffe,

- Energie,

- Produkte,

- Emissionen (Abfall, Abwasser,

Abluft, Abwärme, Lärm).

\section{Umweltziele und Umweltprogramm}

Auf der Grundlage der gemeinsam von Stolzenberg und IÖW aufgestellten Bilanz, der Beurteilung der Umweltrelevanz des unternehmerischen Handelns und der Maßnahmen- 
vorschläge stellte Stolzenberg konkrete Umweltziele und ein Umweltprogramm zur Umsetzung auf. Erwartungsgemäß bezog sich ein großer Teil des Programms auf die Entsorgung. Wie von der Verordnung gefordert, wurden die konkreten, quantitativen Ziele mit Maßnahmen, Zeitrahmen und Zuständigkeiten für die Umsetzung festgelegt. Mit der Einführung des Umweltmanagementsystems wurde das Umweltprogramm später durch organisatorische und Weiterbildungsmaßnahmen ergänzt.

Aufbauend auf den Ergebnissen einer Befragung der Mitarbeiter zu den bestehenden Kommunikationsstrukturen wurden mit der Festlegung der Umweltschutzaufgaben und Zuständigkeiten weitere Bausteine für das Umweltmanagementsystem gelegt. Die Umweltschutzaufgaben sollen die Einhaltung und Umsetzung sowie die regelmäßige Fortschreibung der einzelnen Elemente wie Umweltleitlinien, Ziele und Umweltprogramm gewährleisten - also die Verbesserung des betrieblichen Umweltschutzes. Bestandteile des Umweltmanagementsystems sind die hier bereits genannten Punkte einschließlich der Verfahren zur Prüfung und Beurteilung der Auswirkungen auf die Umwelt und die Erfassung der Rechts- und Verwaltungsvorschriften. Weiter war das Verfahren zur Umweltbetriebsprüfung festzulegen.

Die Verantwortlichkeit für die Anwendung und Aufrechterhaltung des Umweltmanagementsystems hat die Geschäftsführung zu übernehmen, die in der Regel die meisten Aufgaben delegiert. Sie hat die Pflicht, sich Bericht erstatten zu lassen und dafür zu sorgen, daß die Leiter und Mitarbeiter für die ihnen übertragenen Aufgaben auch ausreichend informiert und ausgebildet sind.

\section{Hinreichende Information der Mitarbeiter wichtig}

Nach Anforderung der Öko-Audit-Verordnung müssen Vorkehrungen getroffen werden, die die Sensibilisierung und Ausbildung der Mitarbeiter gewährleisten und eine interne sowie externe Kommunikation über die betrieblichen Umweltaspekte ermöglichen. Vor allem die hinreichende Information der Mitarbeiter im Unternehmen wird häufig - aus Zeit- und Kapazitätsgründen - vernachlässigt. In einer stichprobenartigen Befragung der Mitarbeiter (der Projektgruppe Öko-Audit und Mitarbeiter aus der Produktion) gaben vor allem Mitarbeiter der Produktion an, daß sie an Hintergründen von Umweltschutzmaßnahmen interessiert seien. Die Fachfunktionsträger müssen dagegen für die von ihnen geforderten Aufgaben - z.B. Zuständigkeiten für die Verbesserung der Entsorgung oder Lagerung von wassergefährdenden Stoffen - ausreichende Informationen und Weiterbildungen erhalten. Im Ergebnis wurden im Rahmen eines Maßnahmenplans die geplanten Informationen und Weiterbildungen schriftlich festgehalten und im Umweltprogramm verankert.

Im Umweltmanagementhandbuch - oder einfacher in der Dokumentation - wurden die einzelnen Bestandteile des Umweltmanagementsystems schriftlich festgehalten.

\section{Die Umweltbetriebsprüfung}

Schließlich erfolgte die Abschätzung, ob das Umweltmanagementsystems der Fa. Stolzenberg funktionieren kann und geeignet ist, die selbst gesteckten Umweltleitlinien und -ziele auch einzuhalten bzw. umzusetzen: Dafür wurden zunächst die Prüfungsziele festgelegt, die in der Überprüfung der Funktionsfähigkeit des Umweltmanagementsystems und gegebenenfalls dem Aufzeigen von Verbesserungspotentialen liegen werden. Es mußte ein geeignetes Prüfungsteam zusammengestellt werden, dem zum einen die externen Berater - also das IÖW - angehörten, die über Kenntnis der Anforderungen eines Umweltmanagementsystems und der EG-Öko-Audit-Verordnung verfügen. Weiter waren zwei Mitarbeiterinnen der Fa. Stolzenberg im Team. Auf jeden Fall empfiehlt sich die Teilnahme der für den Umweltschutz zuständigen Mitarbeiter von Unternehmen, da im Rahmen der Prüfung durch die Befragten u. U. wichtige zusätzliche Informationen gegeben werden.

Auf der Grundlage von Dokumenten wie den Umweltvorschriften, den Leitlinien, dem Umweltprogramm und -zielen sowie den spezifischen Gegebenheiten des Betriebes und nach Festlegung der Untersuchungsprioritäten wurden vom IÖW die Fragebögen zur Umweltbetriebsprüfung erstellt. Als wichtigstes Kriterium wurden vom IÖW für die erste Umweltbetriebsprüfung - kurz nach der Einführung des Umweltmanagementsystems - vor allem die Überprüfung der Kenntnis und Einhaltung der Anforderungen der Öko-Audit-Verordnung hinsichtlich der einzelnen Bausteine wie Umweltpolitik, -ziele, -programm und vor allem Umweltschutzorganisation gesehen. Entsprechend wurde die Untersuchungspriorität auch auf die Erfragung der Kenntnis der Umweltschutzaufgaben, der Zuständigkeiten hinsichtlich der Einhaltung der umweltrelevanten Vorschriften, der gesteckten Umweltziele und der Maßnahmen (Umweltprogramm) und Weiterbildung gesetzt.

\section{Endberichte der Berliner Öko-Audit-Modellprojekte}

Die vom IÖW fachlich betreuten Öko-AuditModellprojekte bei den Berliner Firmen Märkisches Landbrot GmbH (Backwaren, $60 \mathrm{Be}$ schäftigte) und der Stolzenberg GmbH (Registratursysteme, 100 Beschäftigte) werden mit der Zertifizierung der Unternehmen im August/September abgeschlossen. Mit den Endberichten ist ab Anfang Oktober zu rechnen.
Nach einer Informations- bzw. Einführungssitzung im Unternehmen mit der Erläuterung von Sinn und Zweck einer solchen Umweltbetriebsprüfung wurde die Prüfung - hauptsächlich in Form von Interviews - durchgeführt. Obwohl das System erst kürzlich mit dem Modellprojekt Öko-Audit bei der Fa. Stolzenberg eingeführt wurde, sind die Ergebnisse schon heute als positiv zu bewerten. Die notwendigen Verbesserungsmaßnahmen entsprechen den bisher festgelegten Umweltzielen und dem Programm des Unternehmens.

Wichtig für die dauerhafte Etablierung des Umweltmanagementsystems ist ein regelmäßiger Informationsaustausch zwischen den Teilnehmern der Projektgruppe Öko-Audit und die Umsetzung des Umweltprogramms. Weiter ist die beabsichtigte regelmäßige Information der übrigen Mitarbeiter für eine Sensibilisierung und effektive Umsetzung der Maßnahmen erforderlich.

\section{Warten auf den externen Gutachter}

Nach der Erstellung der Umwelterklärung wartet die Fa. Stolzenberg - wie auch die Fa. Märkisches Landbrot - auf die Zulassung der Umweltgutachter, damit sie sich von einem externen Gutachter prüfen und schlieBlich zertifizieren lassen können. Die Erfolge des Projektes bei der Fa. Stolzenberg können - neben der erwarteten Zertifizierung - in der zunehmenden Sensibilisierung der Mitarbeiter für den Umweltschutz, der ökologischen Optimierung der Rohund Hilfsstoffbeschaffung und in der Reduzierung des zu entsorgenden Abfallaufkommens durch vermehrtes Recycling gesehen werden. Dabei wird das Unternehmen auch Kosten sparen; die Höhe kann allerdings noch nicht beziffert werden, da die Maßnahmen zur Umsetzung derzeit erst anlaufen.

Sabine Lehmann, Berlin
Diese können über den Berliner Wirtschaftssenat, Martin-Luther-Str. 105, 10825 Berlin gegen Zusendung eines adressierten und mit 4 DM frankierten Rückumschlags (DIN A4Format) bezogen werden. Auch die Umwelterklärungen der Firmen sind ab ca. Mitte September direkt von hier zu beziehen. 
(c) 20I0 Authors; licensee IÖW and oekom verlag. This is an article distributed under the terms of the Creative Commons Attribution Non-Commercial No Derivates License (http://creativecommons.org/licenses/by-nc-nd/3.o/), which permits unrestricted use, distribution, and reproduction in any medium, provided the original work is properly cited. 